Purdue University Purdue e-Pubs

March 2007

\title{
Microfluidic cell fusion under continuous direct current voltage
}

Jun Wang

Chang Lu

Purdue University

Follow this and additional works at: http://docs.lib.purdue.edu/ocspub

Wang, Jun and Lu, Chang, "Microfluidic cell fusion under continuous direct current voltage " (2007). Oncological Sciences Center Publications. Paper 4.

http://docs.lib.purdue.edu/ocspub/4

This document has been made available through Purdue e-Pubs, a service of the Purdue University Libraries. Please contact epubs@purdue.edu for additional information. 


\title{
Microfluidic cell fusion under continuous direct current voltage
}

\author{
Jun Wang and Chang $\mathrm{Lu}^{\mathrm{a})}$ \\ Department of Agricultural and Biological Engineering, Purdue University, 225 South University Street, \\ West Lafayette, Indiana 47907
}

(Received 17 September 2006; accepted 17 October 2006; published online 7 December 2006)

\begin{abstract}
The authors report a technique which produces cell-to-cell electrofusion using a common direct current power supply on a microfluidic platform. In the authors' method, the cells were first conjugated based on biotin-streptavidin interaction. The electrofusion was then conducted by flowing the linked cells through a simple microfluidic channel with geometric variation under continuous direct current voltage. This microfluidics-based technique offers processing at the level of single cell pairs with efficiency comparable to that of conventional electrofusion technique based on electrical pulses. (C) 2006 American Institute of Physics. [DOI: 10.1063/1.2402122]
\end{abstract}

Cell fusion is a powerful tool for analysis of gene expression, chromosomal mapping, antibody production, cloning mammals, and cancer immunotherapy. Cell fusion has been carried out based on several approaches. Chemical and virus-mediated fusion methods were developed in the earlier years based on the application of chemical fusogens such as polyethylene glycol or fusogenic virus (i.e., Sendai). ${ }^{1,2}$ These methods have been associated with limitations such as toxicity to cells, batch-to-batch variability, and low efficiency. In comparison, electrofusion, which is based on the application of high-voltage electric pulses, can be applied to a wide range of cell types with high efficiency and high postfusion viability. ${ }^{3,4}$ Electrofusion typically requires specialized equipment which generates both low-voltage alternating current (ac) for cell alignment/contact and high-voltage direct current (dc) pulses for cell fusion. ${ }^{5}$ Due to the complexity and cost associated with the instrumentation, very few studies have explored realizing this important technique on a microfluidic platform. ${ }^{6,7}$ All these demonstrations involved the application of electrical pulses.

Here, we demonstrate a technique which produces cellto-cell electrofusion using a common dc power supply on a microfluidic platform. In our method, the cells were first conjugated based on biotin-streptavidin interaction. The electrofusion was then conducted by flowing the linked cells through a microfluidic channel with geometric variation under continuous dc voltage. We were able to achieve fusion efficiency comparable to that of conventional specialized equipment based on ac alignment and electrical pulses. The processing was carried out at single cell pair level.

As shown in Fig. 1(a) an electrofusion device in our design consisted of a microfluidic channel with narrow and wide sections. Devices with one or five narrow sections were tested in this work. As we and other researchers previously showed, such channels generate local field intensities which are determined by the channel geometry. ${ }^{8-10}$ Figure 1(b) shows the modeling of the field intensity inside a microfluidic channel with one narrow section when a dc voltage is established across the channel. The modeling suggests that the field strength at the center of the narrow section is around 9.7 times higher than the field strength in the bulk of the wide sections (200 $\mu \mathrm{m}$ or further away from the narrow sec-

\footnotetext{
${ }^{\text {a) }}$ Author to whom correspondence should be addressed; electronic mail: changlu@purdue.edu
}

tion). This number is roughly the ratio between the width in the wide section(s) and the one in the narrow section. In principle we can control the overall voltage so that only the field in the narrow section(s) is high enough for cell fusion and the field in the rest of the channel is too weak to have adverse effects on the viability of cells. When cells flow through the device, they experience field intensity variations equivalent to electrical pulse(s). The "pulse width" is determined by the length of the narrow section and the velocity of cells. The microfluidic chips were fabricated based on polydimethylsiloxane using standard soft lithography method. ${ }^{11}$ We applied Chinese hamster ovary (CHO-K1) cells in our experiments. We demonstrated cell fusion between $\mathrm{CHO}$ cells. In principle the same procedure and device can be applied to fuse two different cell types and obtain hybrid cells as the result. The cells were first chemically linked

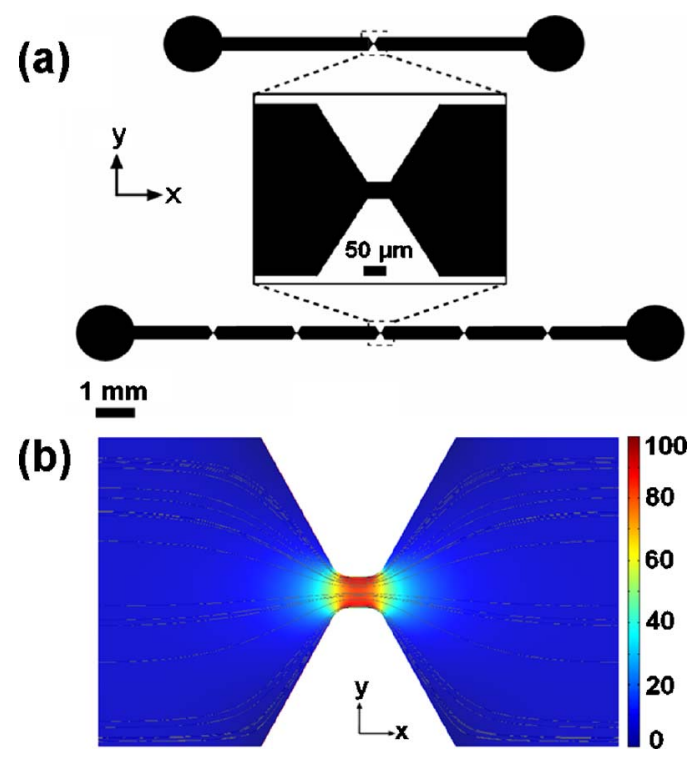

FIG. 1. (Color online) (a) Layout of single-pulsed and five-pulsed microfluidic devices for cell electrofusion. As shown in the inset figure, the narrow section was $50 \mu \mathrm{m}$ long and $40 \mu \mathrm{m}$ wide. The wide sections had a width of $400 \mu \mathrm{m}$. In both devices, the depth of the channel was uniformly $33 \mu \mathrm{m}$. The single-pulsed channel was $8.2 \mathrm{~mm}$ long and the five-pulsed device was $13.2 \mathrm{~mm}$ long. (b) The modeling of the electrical field intensity in a microfluidic structure with alternating wide and narrow sections (COMSOL 3.2). The dimensions of the narrow section and the width of the wide sections were the same as those in (a) and the total length of the structure was $800 \mu \mathrm{m}$. We arbitrarily assigned 100 to the highest field strength in the structure. 

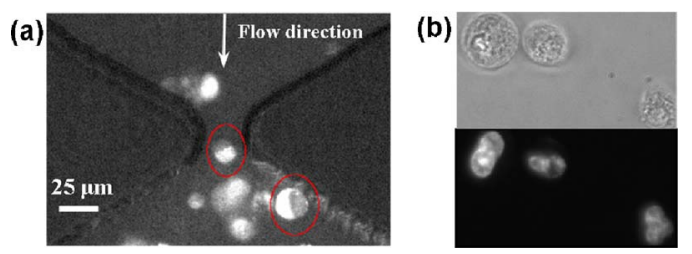

FIG. 2. (Color online) (a) Fluorescent image of fused cells (circled by red, between one cell labeled by calcein AM and one unlabeled) immediately after flowing through the narrow section. The narrow section here was $25 \mu \mathrm{m}$ wide and $30 \mu \mathrm{m}$ long (slightly different from the design in Fig. 1) and the field intensity in the narrow section was $1200 \mathrm{~V} / \mathrm{cm}$. (b) Fused cells labeled by Hoechst 33342. The upper picture is the phase contrast image and the lower picture is the fluorescent image of the same cells. They were generated in a single-pulsed device with $700 \mathrm{~V} / \mathrm{cm}$ in the narrow section and a flow rate of $90 \mu \mathrm{l} / \mathrm{hr}$.

based on the interaction between biotin and streptavidin using a protocol similar to what was reported in the literature. ${ }^{2,13}$ Briefly, the plasma membrane of cells was biotinylated first. One-half of the cell sample was then coated with streptavidin before it was mixed and incubated with the other half of the cell sample. After this step, about 50\%-55\% of the cell population was conjugated. The linked cells had intact membrane between them and there was no exchange of the intracellular contents between different cells. The linked cells were suspended in electrofusion buffer $(1 \mathrm{mM}$ $\mathrm{MgSO}_{4}, 10 \mathrm{mM}$ phosphate buffer, and $250 \mathrm{mM}$ sucrose, $p \mathrm{H}=7.2$ ), then flowed into the electrofusion devices (single pulsed or five pulsed) under the pressure from a syringe pump, while a continuous de voltage was established across the channel. Two approaches were used to observe the cell fusion. First, we labeled half of the cells with a fluorogenic dye, calcein AM (Molecular Probes, Eugene, OR, following the manufacturer's protocol) and left the other half unlabeled before the chemical conjugation. Cell fusion between labeled cells and unlabeled cells was observed immediately after they flowed through the narrow section [Fig. 2(a)]. Calcein (the fluorescent derivative of calcein AM) was observed to diffuse into the other half of the fused cell within minutes. In the second approach, we stained the cell nuclei using a nuclear counterstain, Hoechst 33342 (Molecular Probes, Eugene, OR) and observed the number of nuclei in cells after electrofusion [Fig. 2(b)]. A fraction of the cells contained two or more nuclei. The efficiency of cell fusion is characterized using fusion index (FI) which is defined as the fraction of nuclei in polynucleated cells in the total number of nuclei and is calculated using Eq. (1) below:

$$
\mathrm{FI}(\%)=\frac{\sum_{n=2}^{\infty} n C_{n}}{\sum_{n=1}^{\infty} n C_{n}} \times 100,
$$

where $C_{n}$ is the number of cells containing $n$ nuclei. ${ }^{14} \mathrm{We}$ observed two or three nuclei in the vast majority of the fused cells. It needs to be noted that a fraction of polynucleated cells is due to cell division. Our previous studies showed that the field in the wide sections, which was substantially lower than the threshold for electric breakdown of the membrane, did not affect the cell viability significantly. ${ }^{8}$ We varied the electrofusion field in the narrow section(s) in the experiment. The duration of exposure or the pulse width in the narrow section(s) was also varied by changing the flow rate con- (a)

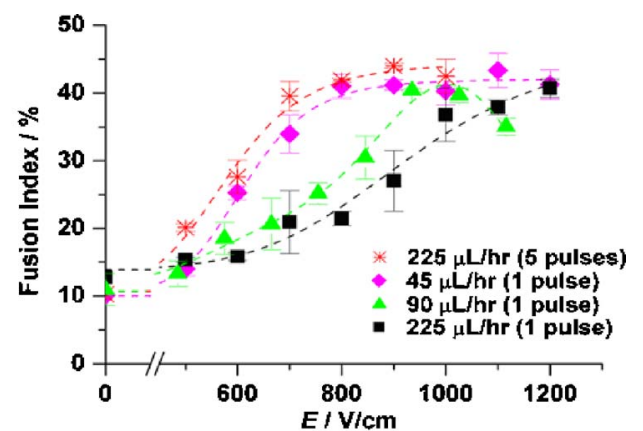

(b)

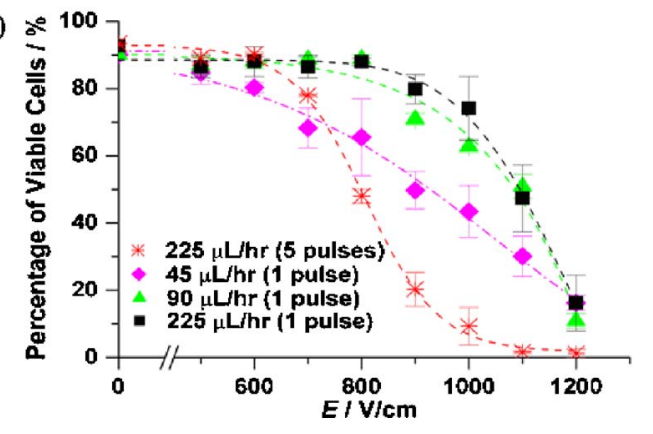

FIG. 3. (Color online) (a) Fusion index (among viable cells) at different electrofusion field strengths and flow rates in the single-pulsed and fivepulsed devices. (b) The percentage of viable cells measured under the same conditions as in (a). Trend lines are added to guide the eye.

trolled by the syringe pump. If we only consider the contribution to the cell velocity from the flow rate of the buffer, the durations for cells to be in the narrow section would be 5.3, 2.6 , and $1.0 \mathrm{~ms}$ when the flow rates are 45, 90, and $225 \mu \mathrm{l} / \mathrm{hr}$, respectively. However, the actual durations (pulse widths) were shorter than the above numbers and varying with the field intensity, due to the contribution to the cell velocity from the electric field. Due to the difficulty in determining the cell velocity in the narrow section(s) experimentally, we specified the pulse widths by indicating the pumping rates (Fig. 3). The fusion index among viable cells was calculated [Fig. 3(a)]. The fusion index was around 10\%$15 \%$ when there was no electric field due to the division in the cell population. ${ }^{15}$ In a single-pulsed device, the fusion index increased remarkably when the field strength in the narrow section became higher during the processing. When the field intensity was increased to $1200 \mathrm{~V} / \mathrm{cm}$, the fusion index was up to $44 \%$ (around 30\% after deducting the fraction due to cell division) at all three flow rates. The pulse width made a significant difference when the field intensity was between 600 and $1000 \mathrm{~V} / \mathrm{cm}$. The longer pulse width (at lower flow rate) resulted in higher fusion efficiency. We also carried out the cell fusion in the five-pulsed device. The application of multiple pulses improved the efficiency of cell fusion significantly. The five-pulsed device yielded fusion indexes that were consistently higher than those that resulted from a single pulse of the same pulse width. The efficiency of cell fusion by our approach was comparable to the result obtained using conventional pulse generator on the same cell type and similar buffer system. ${ }^{15}$ We also examined the viability of cells (both fused and nonfused cells) under these conditions using SYTOX green exclusion ${ }^{8}$ [Fig. 3(b)]. The viability of cells in general decreased with increasing field strength and pulse width. The use of five-pulsed device created a marked decrease in the cell viability. It needs to be noted that the optimum fusion parameters have to be empiri-
AIP license or copyright, see http://apl.aip.org/apl/copyright.jsp 
cally derived for each cell system because they vary considerably from species to species. ${ }^{16}$

Experimental protocols involved in this letter are detailed in an EPAPS document. ${ }^{17}$

We demonstrated that a simple microfluidic device could effectively produce cell fusion under continuous dc voltage, when coupled with chemical linking method. This technique offers efficiency comparable to that of conventional electrofusion technique which requires expensive and complicated apparatus. Cell fusion was conducted at single pair level in the microfluidic channel and the physical dimensions of the channel effectively prevented more than three cells from fusing together. These features are important when cell fusion needs to be conducted based on scarce cell sources such as primary cells. This demonstration will help establish microfluidics as a viable platform for studying a number of important biological problems involving cell fusion. Further studies are needed to characterize the properties of fused cells produced by this technique.

This research was supported by a Purdue Research Foundation fellowship and Purdue University.
${ }^{1}$ J. White, K. Matlin, and A. Helenius, J. Cell Biol. 89, 674 (1981).

${ }^{2}$ R. L. Davidson, K. A. O'Malley, and T. B. Wheeler, Somatic Cell Genet. 2, 271 (1976).

${ }^{3}$ E. Neumann, G. Gerisch, and K. Opatz, Naturwiss. 67, 414 (1980).

${ }^{4}$ U. Zimmermann and J. Vienken, J. Membr. Biol. 67, 165 (1982).

${ }^{5}$ K. L. White, in Methods in Molecular Biology, edited by J. A. Nickoloff (Humana, Totawa, NJ, 1995), Vol. 47, pp. 283-294.

${ }^{6} \mathrm{G}$. Tresset and S. Takeuchi, Biomed. Microdevices 6, 213 (2004).

${ }^{7}$ A. Stromberg, A. Karlsson, F. Ryttsen, M. Davidson, D. T. Chiu, and O. Orwar, Anal. Chem. 73, 126 (2001).

${ }^{8}$ H. Y. Wang and C. Lu, Anal. Chem. 78, 5158 (2006).

${ }^{9}$ S. C. Jacobson, C. T. Culbertson, J. E. Daler, and J. M. Ramsey, Anal. Chem. 70, 3476 (1998).

${ }^{10}$ M. L. Plenert and J. B. Shear, Proc. Natl. Acad. Sci. U.S.A. 100, 3853 (2003).

${ }^{11}$ D. Duffy, J. McDonald, O. Schueller, and G. Whitesides, Anal. Chem. 70, 4974 (1998).

${ }^{12}$ T. C. Bakker Schut, Y. M. Kraan, W. Barlag, L. de Leij, B. G. de Grooth, and J. Greve, Biophys. J. 65, 568 (1993).

${ }^{13}$ M. Tomita and T. Y. Tsong, Biochim. Biophys. Acta 1055, 199 (1990).

${ }^{14}$ G. Poste, and D. Papahadjopoulos, Methods Cell Biol. 14, 23 (1976).

${ }^{15}$ C. Ramos, D. Bonenfant, and J. Teissie, Anal. Biochem. 302, 213 (2002).

${ }^{16}$ U. Zimmermann, P. Scheurich, G. Pilwat, and R. Benz, Angew. Chem., Int. Ed. Engl. 20, 325 (1981).

${ }^{17}$ See EPAPS Document No.E-APPLAB-89-205649 for the simulation and experimental protocols involved in this study. The document can be reached via a direct link in the online article's HTML reference section or via the EPAPS homepage (http://www.aip.org/pubservs/epaps.html/. 